\title{
Optimization of Aloe vera and alginate as encapsulating matrices for Lactobacillus acidophilus using FCCD-RSM approach
}

\begin{abstract}
In order to maintain the viability of probiotic, the used of prebiotic is important. Aloe vera has been hypothesized to be a prebiotic potential for probiotic. Due to low viability of encapsulated cells, Aloe vera gel was used to be part of enacapsulating matrices for probioctic, Lactobacillus acidophilus. As a result, optimization of Aloe vera gel with sodium alginate was carried out in order to determine the maximum encapsulation yield. Face centered composite design-response surface methodology (FCCD-RSM) was employed to optimize the sodium alginate concentration and Aloe vera gel (prebiotic) composition during microencapsulation process in performing a better encapsulation yield for L. acidophilus. The encapsulation yield (EY) of encapsulated L. acidophilus was investigated with respect to two variables which were sodium alginate (X1) and Aloe vera gel (X2). Result obtained with polynomial regression model used in this study showed highly significant (0.0013) with R2 value was 0.9138. Aloe vera gel was showed highly significant $(\mathrm{p}<0.05)$ effect to the encapsulation yield. The optimum Aloe vera gel and sodium alginate were obtained at $1.12 \%$ $(\mathrm{v} / \mathrm{v})$ and $1.28 \%(\mathrm{w} / \mathrm{v})$, respectively. Based on the verification process, the experimental and predicted results were showed not significant difference $(\mathrm{p}<0.05)$.
\end{abstract}

Keyword: Optimization; RSM; Encapsulation; Lactobacillus acidophilus; Alginate; Aloe vera gel 